Viding, E; Seara-Cardoso, A; (2013) Why do children with disruptive behavior disorders keep making bad choices? [Editorial comment]. American Journal of Psychiatry, 170 (3) 253 - 255.

10.1176/appi.ajp.2012.12121551. Downloaded from UCL Discovery:

http://discovery.ucl.ac.uk/1390058.

ARTICLE

\title{
Why do children with Disruptive Behavior Disorders keep on making bad choices?
}

\author{
Essi Viding, $P h D^{*} \&$ Ana Seara Cardoso, MSc \\ Developmental Risk and Resilience Unit, Division of Psychology and Language Sciences, \\ University College London, United Kingdom \\ * Corresponding Author: Essi Viding, Division of Psychology and Language Sciences, \\ University College London, 26 Bedford Way, London WC1H OAP, UK; Telephone: +44 (0) \\ 2076795874; E-mail: e.viding@ucl.ac.uk.
}

Disruptive Behavior Disorders (DBDs), such as Conduct Disorder and Oppositional Defiant Disorder, are common reasons for a childhood referral to mental health and educational services and represent a substantial public health cost (1). Children with DBDs are not only at risk of developing antisocial personality disorder in adulthood, but they are also at risk of developing a number of other psychiatric and physical health problems $(2,3)$. What is striking for people who come to contact with these children, either in a research or clinical/educational settings, are the repeated poor choices they make. Children with DBDs often act impulsively, with apparently little ability to compute the consequences of their poor behavioral choices. Many of these children desperately want to make better choices, but are upset as they find themselves, yet again, in trouble that they did not see coming. Others, may pursue selfish goals, but with overly optimistic predictions of the likely outcome of their actions and experience subsequent frustrated aggression when things do not work out. In order to adequately formulate and target interventions that will help children with DBDs we need to better understand the information processing abnormalities that characterize their decision-making.

Behavioral studies have demonstrated that children with DBDs show impairments in standard learning and reversal learning tasks (4). A handful of neuroimaging studies have also documented atypical neural responses to reward-punishment learning in children with DBDs, for example in the orbitofrontal cortex and caudate (e.g. 5, 6 ). In this issue of the Journal, White et al. (7) report the first ever study to examine the neural responses of children with DBDs (as compared with typically developing children) during decision-making and reinforcement, using model-based fMRI. Successful decision-making involves two critical components: (i) the appropriate representation of reinforcement expectancies (i.e. the expected value [EV] associated with a stimulus/action); and (ii) prediction error [PE] signaling (i.e. signal regarding the difference between the actual and the expected reward/punishment that enables updating of the reinforcement expectancies). PE can be positive, when the actual value is better than expected, or negative, when the actual value is worse than expected. White et al. (7) evaluated EV processing during the decision-making phase and PE processing during the feedback phase of a probabilistic version of the passive-avoidance decision-making paradigm. They showed that, compared with typically developing children, children with DBDs showed significantly reduced modulation of activity by EV within the ventromedial prefrontal cortex when choosing objects and within the anterior insula when refusing objects during this task, indicating disrupted EV signaling. 
White et al. (7) also demonstrated that in the caudate there was reduced modulation of activity by PE to reward, but increased modulation of activity to punishment in children with DBDs, indicating disrupted PE signaling that appeared qualitatively different to that, which was seen in typically developing youth.

These are important findings that have bearing on both theoretical accounts of DBDs, as well as translational efforts for these conditions. White et al. (7) data suggest that a simple deficit model will not capture the decision-making impairment apparent in children with DBDs. Instead, they argue that we may be seeing a fundamentally different organization of information processing system responsible for reward-punishment learning and appropriate decision-making. The study by White et al. (7) thus demonstrates that children with DBDs fail to appropriately modulate brain responses in the face of negative PEs. The authors suggest that the atypical neural modulation by EV and PE indexes increased risk for antisocial behavior in children with DBDs. It is clear that appropriate EV signaling is critical in making good behavioral choices and that appropriate PE signaling is important for the individual's ability to generate further, more accurate EVs. White et al. (7) argue that disrupted EV and PE signaling will lead to the selection of poor behavioral choices and (given certain environmental contexts) antisocial behavior.

Of course, the study has some limitations that future research could helpfully address. This was the first study of its kind and examined EV and PE learning cross-sectionally. The authors appear to suggest that poor PE learning will lead to inappropriate EV signaling over time. However, it also plausible that inappropriate EV signaling will, in part, contribute to atypical patterns of PE over time. It would be interesting to study development of both EV and PE processing longitudinally using cross-lag models - in both typically developing children, as well as children with DBDs. Another consideration for the future concerns the type of reward used to study EV and PE processing in children with DBDs. A specific type of reward (money; although it was not clear from the paper whether the money was actually awarded to the children) was used in this study. In the future, it might be helpful to map rewards that hold high subjective value to each participant and use these to study EV and PE. In other words, are there differences in EV and PE processing across the categories of reward, or does the subjective salience of the reward modulate the extent of EV and PE processing abnormalities that we see in children with DBDs? Currently, it is unclear whether the subjective, motivational value of the rewards was equal to all participants and whether this contributed to the group differences. Finally, the authors themselves note is that "Currently, it is unknown what might cause such a fundamental reorganization of PE punishment signaling." We know that DBDs are moderately to strongly heritable (8). We also know that parents of children with DBDs not only pass on a degree of genetic vulnerability to their children, but can also provide a poor parenting environment (8). A wealth of research now indicates that the parenting environments of children with DBDs typically involve less positive and more negative reinforcement, as well as less consistent reinforcement contingencies than the parenting environments of typically developing children. It may thus be that there are children who have the unfortunate 'double-whammy' of being genetically vulnerable to atypical EV and PE processing and receiving childhood learning environments that further derail EV and PE processing. Genetically informative, longitudinal study designs would be helpful in investigating the etiology of the EV and PE processing in children with DBDs.

In sum, White et al. (7) have made an important contribution to the literature probing the neural correlates of specific decision-making impairments that correlated with DBDs. Their study suggests that a simple deficit model of decision-making is not appropriate for describing the information processing profile of children with DBDs. We look forward to future work that both replicates and extends this research and particularly welcome the promise of this research in informing interventions to help children with DBDs. Treatment efforts for DBDs often involve behavior modification programs that rely on rewards and 
punishments. It will be of considerable interest to build on the findings of this study in order to develop a more precise understanding of the specific reinforcement processing abnormalities in children with DBDs, and how these might be best modified with appropriate cognitive-behavioral manipulations.

Essi Viding receives grant support from the Economic and Social Research Council (RES062-23- 2202). Ana Seara-Cardoso receives PhD funding from the Portuguese Foundation for Science and Technology (Fundação para a Ciência e Tecnologia; SFRH/BD/60279/2009).

\section{References:}

1. Scott S, Knapp M, Henderson, J, Maughan, B. Financial cost of social exclusion: follow up study of antisocial children into adulthood. BJM. 2001; 323:1-5

2. Kim-Cohen J, Caspi A, Moffitt TE, Harrington HL, Milne BJ, Poulton R. Prior juvenile diagnoses in adults with mental disorder: developmental follow-back of a prospectivelongitudinal cohort. Arch Gen Psychiatry. 2003; 60:709-17

3. Odgers CL, Capsi A, Broadbent JM, et al. Prediction of differential adult health burden by conduct problem subtypes in males. Arch Gen Psychiatry. 2007; 64:476-84

4. Blair, JR. Neuroimaging of psychopathy and antisocial behavior: a targeted review. Curr Psychiatry Rep. 201; 12(1):76-82.

5. Finger EC, Marsh AA, Blair KS, Reid ME, Sims C, Ng P, et al. Disrupted reinforcement signaling in the orbitofrontal cortex and caudate in youths with conduct disorder or oppositional defiant disorder and a high level of psychopathic traits. Am J Psychiatry. 2011 Feb; 168(2):152-62.

6. Rubia K, Smith AB, Halari R, Matsukura F, Mohammad M, Taylor E, et al. Disorderspecific dissociation of orbitofrontal dysfunction in boys with pure conduct disorder during reward and ventrolateral prefrontal dysfunction in boys with pure ADHD during sustained attention. Am J Psychiatry. 2009;166(1):83-94.

7. White S, Pope K, Sinclair S, Fowler K,, Brislin S, Williams W, et al. Disrupted expected value and prediction error signaling in youth with disruptive behavior disorders during a passive avoidance task. Am J Psychiatry, XXXXXXX.

8. Moffitt TE. The new look of behavioral genetics in developmental psychopathology: geneenvironment interplay in antisocial behaviors. Psychol Bull. 2005 Jul;131(4):533-54. 